\title{
Lysyl oxidase-like 2 is expressed in kidney tissue and is associated with the progression of tubulointerstitial fibrosis
}

\author{
SUNG-EUN CHOI ${ }^{1}$, NARA JEON ${ }^{1}$, HOON YOUNG CHOI ${ }^{2,3}$, JAE IL SHIN ${ }^{3,4}$, \\ HYEON JOO JEONG ${ }^{1,3}$ and BEOM JIN LIM ${ }^{1,3}$ \\ Departments of ${ }^{1}$ Pathology and ${ }^{2}$ Internal Medicine; ${ }^{3}$ Institute of Kidney Disease Research; ${ }^{4}$ Department \\ of Pediatrics, Yonsei University College of Medicine, Seoul 03722, Republic of Korea
}

Received December 22, 2016; Accepted May 5, 2017

DOI: $10.3892 / \mathrm{mmr} .2017 .6918$

\begin{abstract}
Tubulointerstitial fibrosis is a common end point of chronic kidney diseases, and preventing its progression is key to avoiding renal failure. Transforming growth factor- $\beta$ (TGF- $\beta$ ) and associated molecules promote tubulointerstitial fibrosis; however, effective therapies targeting these molecules have yet to be developed. Lysyl oxidase-like 2 (LOXL2), which is involved in invasive growth and metastasis of malignant neoplasms, has recently been reported to serve a key role in hepatic and pulmonary fibrosis. However, little is currently known regarding LOXL2 expression in the kidney and its involvement in tubulointerstitial fibrosis. The present study evaluated LOXL2 expression in human and mouse kidney tissues, as well as in cultured renal cells. LOXL2 protein expression was detected in glomerular capillary loops and tubular epithelial cells in human and mouse kidneys. Glomerular LOXL2 was localized to the cytoplasm of podocytes, as determined by double immunofluorescence microscopy using a podocyte marker (synaptopodin). This result was supported by western blot analysis, which demonstrated that LOXL2 protein expression is present in cultured human podocytes and HK-2 human proximal tubular cells. In addition, the mRNA and protein expression levels of LOXL2 were higher in a mouse model of tubulointerstitial fibrosis compared with in control mice. In addition, immunohistochemistry results demonstrated that LOXL2 is present in the fibrous interstitium and infiltrating mononuclear cells in a mouse model of tubulointerstitial fibrosis. The present study demonstrated that LOXL2 is expressed in compartments of renal tissue, where it appears to contribute to the progression of tubulointerstitial fibrosis.
\end{abstract}

Correspondence to: Dr Beom Jin Lim, Department of Pathology, Yonsei University College of Medicine, 50-1 Yonsei-ro, Seodaemoon-gu, Seoul 03722, Republic of Korea

E-mail: bjlim@yuhs.ac

Key words: lysyl oxidase-like 2, tubulointerstitial fibrosis, transforming growth factor- $\beta$, podocyte

\section{Introduction}

Tubulointerstitial fibrosis is a common end point of diabetic nephropathy, allograft rejection and various glomerulonephritides. Therefore, preventing tubulointerstitial fibrosis is an important strategy in the treatment of chronic kidney diseases.

Members of the lysyl oxidase (LOX) family [e.g., LOX and LOX-like (LOXL)1-4] are responsible for the cross-linking of collagen and elastin in the extracellular matrix through their copper-dependent amine oxidase activity. In addition, LOX family members exhibit various functions in cell proliferation, tumor invasion and metastasis, and organ development (1). LOXL2 is the most thoroughly studied of the LOX family members. Its expression is associated with tumor cell differentiation in colon and esophageal carcinoma (2), and proliferation, migration and invasion of human hepatocellular carcinoma cells (3). Increased LOXL2 expression is also associated with poor survival in squamous cell carcinoma of the larynx and lung (4), and appears to serve a role in the metastatic potential of breast (5) and gastric carcinoma (6). Potential mechanisms underlying the effects of LOXL2 include fibroblast activation in the tumor microenvironment (7), induction of epithelial-mesenchymal transition in tumor cells (5), and matrix remodeling via regulation of tissue inhibitor of metalloproteinase-1 and matrix metalloproteinase-9 (8).

The contribution of LOXL2 to benign fibrosing diseases has been studied in several organs. Previous studies have indicated that LOXL2 expression is associated with hepatic fibrosis in Wilson's disease, primary biliary cirrhosis (9) and hepatocellular carcinoma (10). Elevated serum LOXL2 levels are also associated with disease progression in idiopathic pulmonary fibrosis (11), and LOXL2 upregulation is associated with scar formation following glaucoma surgery (12).

An inhibitory monoclonal antibody to LOXL2 has been developed; AB0023 binds to human and mouse LOXL2, and AB0024 (simtuzumab) is its humanized form. The antifibrotic effects of AB0023, AB0024 and other inhibitory antibodies have been determined in several organs. For example, in a rabbit model of glaucoma surgery, AB0023 attenuated postoperative fibrosis (12). In BALB/c mice, AB0023 attenuated tetrachloride-induced hepatic fibrosis and decreased phosphorylated-Smad3 signaling. In C57BL/6 mice, AB0023 attenuated high-dose bleomycin-induced pulmonary fibrosis, 
and this effect was mediated by inhibiting fibroblast recruitment and activation (13). Based on the results of previous animal experiments, clinical trials of simtuzumab for human fibrosing diseases have been performed. The target diseases include advanced liver fibrosis due to human immunodeficiency virus and hepatitis $\mathrm{C}$ virus infection (14), and idiopathic pulmonary fibrosis (clinicaltrials.gov/ct2/show/NCT01769196).

Although fibrosis is a clinically important pathological process in kidney disease, little is currently known regarding the expression of LOXL2 in renal tissue and its contribution to the development of renal tubulointerstitial fibrosis. In the present study, the expression of LOXL2 in normal kidney was evaluated in tissues and cell lines. In addition, to evaluate its possible profibrotic role, LOXL2 expression was evaluated in the kidneys of mice following the induction of tubulointerstitial fibrosis.

\section{Materials and methods}

Renal cell culture. For the in vitro study, human podocytes and the most widely used proximal and distal tubular epithelial cell lines were selected. Immortalized human proximal tubular cells (HK-2 cells) were purchased from the American Type Culture Collection (Manassas, VA, USA) and canine tubular cells (MDCK cells; cat. no. 10034) were purchased from the Korean Cell Line Bank (Seoul, South Korea). HK-2 cells were cultured in Dulbecco's modified Eagle's medium (DMEM)/Nutrient Mixture F-12 (Gibco; Thermo Fisher Scientific, Inc., Waltham, MA, USA) and $10 \%$ fetal bovine serum (FBS; Gibco; Thermo Fisher Scientific, Inc.). MDCK cells were cultured in DMEM (Gibco; Thermo Fisher Scientific, Inc.). Conditionally immortalized human podocytes were provided by Dr Moin A. Saleem (University of Bristol, Bristol, UK) and Dr Jun Oh (University Medical Center Hamburg-Eppendorf, Hamburg, Germany). The podocytes were grown in RPMI-1640 medium (Gibco; Thermo Fisher Scientific, Inc.), 10\% FBS and insulin-transferrin-selenium supplement (Gibco; Thermo Fisher Scientific, Inc.) at $33^{\circ} \mathrm{C}$ to activate the SV40 large $\mathrm{T}$ antigen. The cells were then cultured at $37^{\circ} \mathrm{C}$ for 2 weeks to induce differentiation (15), which was confirmed by western blotting for synaptopodin (data not presented).

Animal model of tubulointerstitial fibrosis. Male CD1 mice of 8 weeks of age (Orient Bio, Inc., Seongnam, South Korea) were used for the animal experiments. Mice were housed at $20^{\circ} \mathrm{C}$ with a 12-h light/dark cycle and free access to rodent chow and water. Tubulointerstitial fibrosis was induced in 4 mice (mean body weight, $42.5 \mathrm{~g}$ ) by intraperitoneal injection of folic acid (240 $\mu \mathrm{g} / \mathrm{g}$ body weight), according to previously described methods $(16,17)$. The folic acid solution was prepared by dissolving folic acid powder (Sigma-Aldrich; Merck KGaA, Darmstadt, Germany) in $0.3 \mathrm{M} \mathrm{NaHCO}_{3}$. Control CD1 mice $(\mathrm{n}=4$; mean body weight, $43.1 \mathrm{~g})$ were intraperitoneally injected with the same amount of vehicle $\left(\mathrm{NaHCO}_{3}\right)$. After 4 weeks, the mice were sacrificed, and the kidneys were harvested. Fresh frozen tissues were stored at $-70^{\circ} \mathrm{C}$ subsequent to instant freezing in liquid nitrogen. Additional tissues were fixed in $4 \%$ formaldehyde for $24 \mathrm{~h}$ at room temperature and embedded in paraffin overnight at $55-65^{\circ} \mathrm{C}$ using an automatic tissue processer (EFTP-FAST 360; Intelsint, Turin, Italy). The present study was approved by the Institutional Animal
Care and Use Committee of Yonsei University Health System (Seoul, South Korea).

Western blot analysis of LOXL2 expression. HK-2 cells, MDCK cells and differentiated human podocytes were lysed in radioimmunoprecipitation assay buffer (Biosesang, Inc., Seongnam, Korea) containing protease inhibitor cocktail (Roche Diagnostics, Indianapolis, IN, USA). The samples were centrifuged at $15,871 \mathrm{x}$ g for $30 \mathrm{~min}$ at $4^{\circ} \mathrm{C}$, and protein concentration was measured using the bicinchoninic acid protein assay kit (Thermo Scientific, Inc.) according to the manufacturer's protocol. Protein samples $(50 \mu \mathrm{g})$ were separated by $10 \%$ SDS-PAGE for $2 \mathrm{~h}$ at $100 \mathrm{~V}$ and were then transferred to a polyvinylidene fluoride membrane. After blocking with $3 \%$ skim milk for $1 \mathrm{~h}$ at room temperature, the membrane was incubated with the following primary antibodies overnight at $4^{\circ} \mathrm{C}$ : Anti-synaptopodin (cat. no. sc-21537; 1:2,000; Santa Cruz Biotechnology, Inc., Dallas, TX, USA), anti-LOXL2 (cat. no. ab96233; 1:500; Abcam, Cambridge, MA, USA) and anti-LOXL2, which has epitope homology to canine species (cat. no. TA335061; 1:100; OriGene Technologies, Inc., Rockville, MD, USA). The membrane was then washed with Tris-buffered saline containing $0.1 \%$ Tween-20 and was incubated with horseradish peroxidase-labeled secondary antibodies (cat. no. sc-2020; 1:5,000; Santa Cruz Biotechnology, Inc.; and cat. no. K4003; 1:5,000; Dako; Agilent Technologies, Inc., Santa Clara, CA, USA) for $1 \mathrm{~h}$ at room temperature. Protein bands were visualized using Pierce Enhanced Chemiluminescence Western Blotting Substrate (Thermo Fisher Scientific, Inc.). After stripping the membrane with Restore Western Blot Stripping Buffer (Thermo Fisher Scientific, Inc.) for $15 \mathrm{~min}$ at room temperature, the membrane was incubated with an anti- $\beta$-actin antibody (cat. no. sc-47778; 1:2,000; Santa Cruz Biotechnology, Inc.), which was used as a loading control. In addition, fresh frozen kidneys from vehicle or folic acid-injected mice were homogenized, and western blotting was performed in a similar manner. Semi-quantification of the bands was performed by densitometry using Image $\mathbf{J}$ software (version 1.50i; National Institutes of Health, Bethesda, MD, USA).

Immunohistochemistry and immunofluorescence analysis of LOXL2 in human and mouse kidneys. LOXL2 expression in human and mouse kidneys was evaluated by immunohistochemistry and Olympus BX53 light microscope (Olympus Corporation, Tokyo, Japan). Paraffin-embedded human kidney tissues from 4 patients were obtained from the surgical pathology archive of the Department of Pathology, Yonsei University, Gangnam Severance Hospital (Seoul, South Korea). These tissues were obtained from a non-neoplastic portion of a nephrectomy specimen of a renal tumor. The use of archived human tissue was approved by the institutional review board of Yonsei University, Gangnam Severance Hospital. The paraffin-embedded kidneys from the aforementioned vehicle- and folic acid-injected mice were also used for immunohistochemical analysis of LOXL2 expression.

Human and mouse kidney tissues were cut into 4- $\mu \mathrm{m}$ sections, deparaffinized, and rehydrated using xylene and ethanol. Antigen retrieval was conducted by microwaving the tissue sections in $0.01 \mathrm{M}$ sodium citrate buffer ( $\mathrm{pH}$ 6.0) for 
$10 \mathrm{~min}$. Endogenous peroxidase activity was blocked with $0.3 \%$ hydrogen peroxidase for $10 \mathrm{~min}$. The tissue sections were then incubated overnight with a primary antibody against LOXL2 (cat. no. ab96233; 1:1,000; Abcam) at $4^{\circ} \mathrm{C}$, followed by incubation with a horseradish peroxidase-labeled secondary antibody (cat. no. K4003; prediluted; Dako; Agilent Technologies, Inc.) for $1 \mathrm{~h}$ at room temperature. The protein was visualized using the chromogen diaminobenzidine.

Double immunofluorescence staining for LOXL2 along with synaptopodin was performed in a similar manner. Sections were incubated with the primary antibody against LOXL2 (1:100) for $4 \mathrm{~h}$ at room temperature, followed by incubation with a Texas Red-conjugated anti-rabbit immunoglobulin G (cat. no. TI-1000; 1:50; Vector Laboratories, Inc., Burlingame, CA, USA) overnight at $4^{\circ} \mathrm{C}$. Subsequently, the tissue sections were incubated with a primary antibody against synaptopodin (cat. no. 65294; 1:50; Progen Biotechnik GmbH, Heidelberg, Germany) for $4 \mathrm{~h}$ at room temperature, followed by incubation with fluorescein isothiocyanate-conjugated anti-mouse secondary antibody (cat. no. FI-2000; 1:50; Vector Laboratories, Inc.) overnight at $4^{\circ} \mathrm{C}$.

Reverse transcription-quantitative polymerase chain reaction (RT-qPCR) analysis of LOXL2 expression. The mRNA expression levels of LOXL2 in renal cells and fresh frozen kidneys from the vehicle- or folic acid-injected mice were analyzed by RT-qPCR. RNA was extracted using the RNeasy Mini kit (Qiagen GmbH, Hilden, Germany) according to the manufacturer's protocol, and the RNA was reverse transcribed using the QuantiTect Reverse Transcription kit (Qiagen GmbH) according to the manufacturer's protocol. PCR amplification was performed using TaqMan Gene Expression Master Mix and an ABI 7900 HT real-time PCR system (Applied Biosystems; Thermo Fisher Scientific, Inc.) with the following thermal cycle: $2 \mathrm{~min}$ at $50^{\circ} \mathrm{C}$ for uracil DNA-glycosylase enzyme incubation and $10 \mathrm{~min}$ at $95^{\circ} \mathrm{C}$ for AmpiTaq Gold enzyme activation, followed by 40 cycles of $15 \mathrm{sec}$ at $95^{\circ} \mathrm{C}$ and $1 \mathrm{~min}$ at $60^{\circ} \mathrm{C}$. TaqMan primer/probes for mouse LOXL2 (cat. no. Mm00804740_m1) and ribosomal 18S RNA (cat. no. Mm03928990_g1) were purchased from Applied Biosystems (Thermo Fisher Scientific, Inc.). Expression was calculated using the $2^{-\Delta \Delta C q}$ method (18).

Statistical analysis. Quantitative analysis was performed for the western blotting and RT-qPCR results. Folic acid-injected and vehicle-treated control groups were compared ( $\mathrm{n}=4$ mice/group). Data are expressed as the mean \pm standard deviation and were compared using the Mann-Whitney $U$ test. The analyses were performed using GraphPad Prism 6 for Windows (GraphPad Software, Inc., La Jolla, CA, USA). P<0.05 was considered to indicate a statistically significant difference.

\section{Results}

LOXL2 protein expression in human and mouse kidneys. Immunohistochemistry results demonstrated that LOXL2 protein was expressed in the glomeruli and tubular epithelial cells in human kidneys (Fig. 1A). In the glomeruli, LOXL2 staining was observed along the outer surface of capillary loops. In the tubular epithelial cells, LOXL2 staining was cytoplasmic
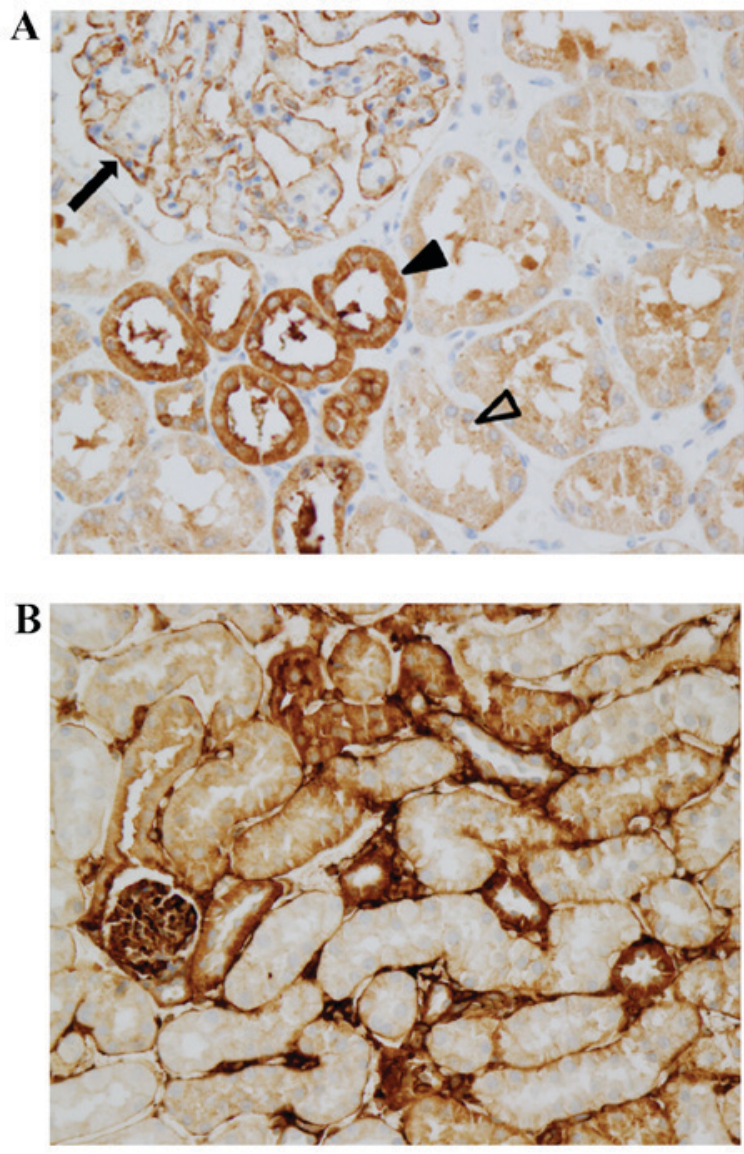

Figure 1. LOXL2 expression in human and mouse kidneys. Results of immunohistochemistry revealed that LOXL2 expression is present in glomeruli and tubular epithelial cells in the (A) human and (B) mouse kidney samples. In the glomeruli, LOXL2 staining was observed along glomerular capillary loops (arrow). In the tubular epithelial cells, LOXL2 staining was observed in the cytoplasm (arrow heads). The staining was more prominent in distal tubules (black arrow head) than in proximal tubules (open arrow head). Magnification, x400. LOXL2, lysyl oxidase-like 2.

with no nuclear or membranous staining observed (Fig. 1A). Proximal and distal tubules expressed LOXL2; however, more prominent staining was detected in distal tubular epithelial cells. In the mouse kidney, LOXL2 staining was also observed in the glomeruli and tubular epithelial cells (Fig. 1B).

To determine the precise location of LOXL2 expression, double immunofluorescence microscopy, using the podocyte marker synaptopodin, was performed in the human kidney. LOXL2 expression was detected in the cytoplasm of podocytes, where synaptopodin expression was also observed (Fig. 2).

LOXL2 expression in cultured cells. In cultured cell lines, LOXL2 expression was detected in human podocytes and HK-2 cells, as determined by western blot analysis, which supported the aforementioned results. However, MDCK cells, which are tubular cells derived from canine kidney, did not express LOXL2 (Fig. 3).

LOXL2 expression in a mouse model of tubulointerstitial fibrosis. Folic acid injection successfully induced diffuse renal tubulointerstitial fibrosis in mice (Fig. 4A and B). Immunohistochemistry analysis of LOXL2 demonstrated strong immunoreactivity in infiltrating inflammatory cells and 

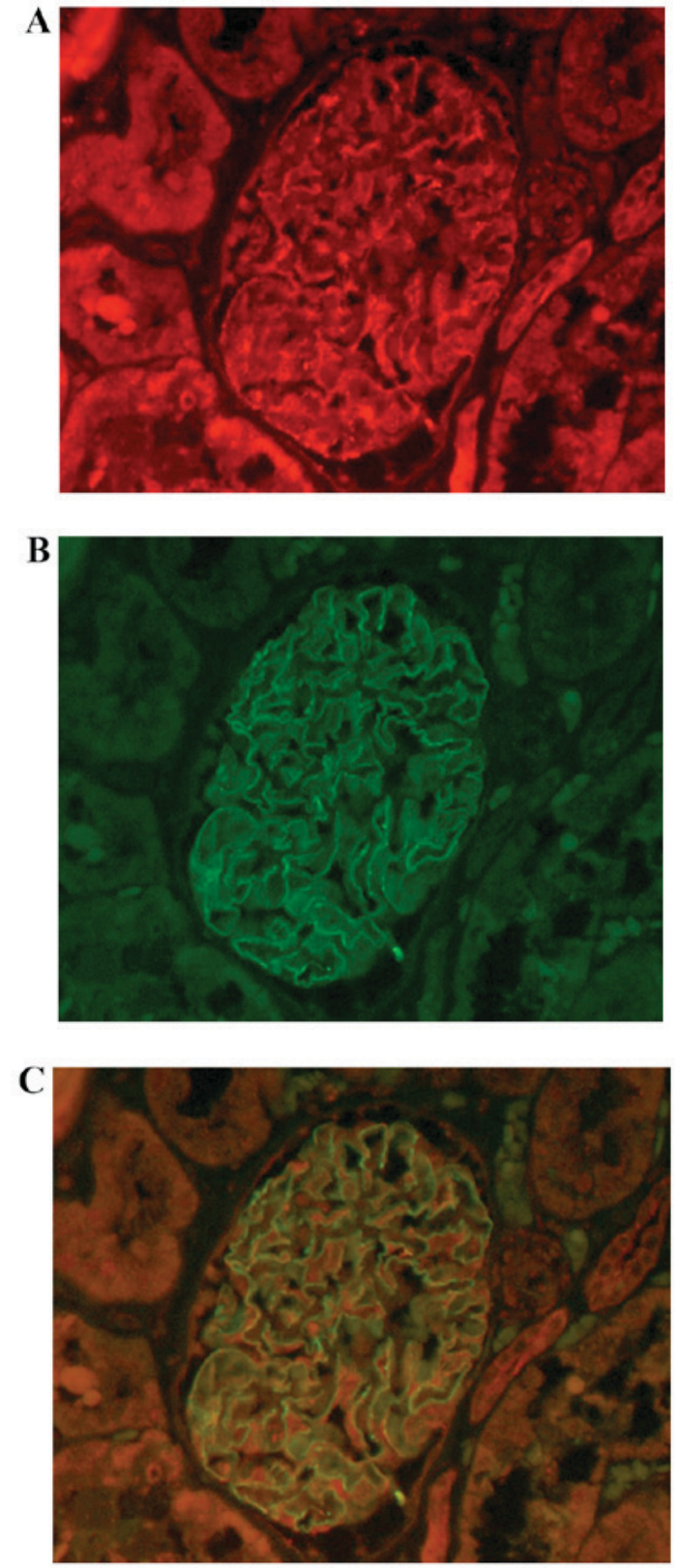

Figure 2. Double immunofluorescence microscopy analysis of LOXL2 in human podocytes. In the glomeruli, (A) LOXL2 and (B) the podocyte-specific marker synaptopodin were (C) colocalized in podocyte cytoplasm. Magnification, x400. LOXL2, lysyl oxidase-like 2.

the interstitium, in addition to glomerular and tubular expression (Fig. 4C). RT-qPCR analysis indicated that the mRNA expression levels of LOXL2 were significantly increased in folic acid-injected mice compared with vehicle-injected controls ( $\mathrm{P}=0.029$; Fig. 4D). In addition, as determined by western blotting, the protein expression levels of LOXL2 were increased in folic acid-injected mice compared with vehicle-injected mice $(\mathrm{P}=0.023$; Fig. $4 \mathrm{E})$.

\section{Discussion}

Tubulointerstitial fibrosis occurs during the progression of all chronic kidney diseases. Preventing tubulointerstitial fibrosis, regardless of its etiology, is important in the preservation of renal function. TGF- $\beta$ is a key molecule in the development

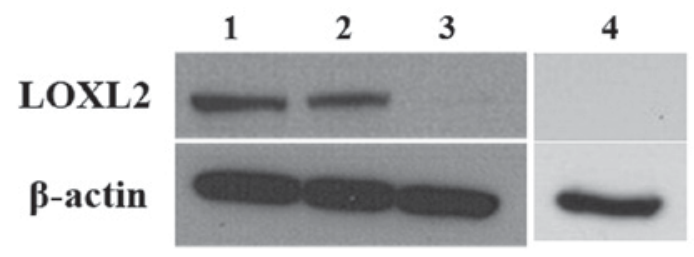

Figure 3. Western blot analysis of LOXL2 expression in cultured proximal tubular cells and podocytes. LOXL2 protein levels in cultured human proximal tubular cells (HK-2), canine tubular cells (MDCK) and immortalized human podocytes were evaluated by western blotting. The results demonstrated that LOXL2 is expressed in HK-2 cells and podocytes, but not in MDCK cells. Another group of MDCK cells was reacted with canine LOXL2 antibody and exhibited no expression. Lane 1, human podocyte; lane 2, HK-2 cell; lane 3, MDCK cell; lane 4, MDCK cell (canine antibody). LOXL2, lysyl oxidase-like 2.

and progression of tubulointerstitial fibrosis. Activators of latent TGF- $\beta$ include integrin $\alpha v \beta 6$; downstream signal transduction molecules of the TGF- $\beta$ signaling pathway, such as members of the Smad family and mitogen-activated protein kinases; and upstream signal transduction molecules. Contributing signal transduction pathways and mechanisms include the Wnt/ $\beta$-catenin pathway, platelet-derived growth factors, epithelial-mesenchymal transition and autophagy (19). Recently, knowledge regarding the mechanisms underlying the pathogenesis of tubulointerstitial fibrosis has increased, and an animal study regarding its prevention has produced promising results (20). However, few therapeutic agents for clinical use have been developed. Therefore, a better understanding of the molecular mechanisms is required for the development of novel treatment strategies for tubulointerstitial fibrosis.

LOXL2, which serves a role in cancer metastasis, is also involved in organ fibrosis. Previous studies have demonstrated the involvement of LOXL2 in hepatic and pulmonary fibrosis, and clinical trials have evaluated a LOXL2-specific inhibitor (9,11-13). Although the clinical implications of kidney fibrosis are substantial, little is currently known regarding LOXL2 expression in this organ, and the role of LOXL2 in tubulointerstitial fibrosis remains to be elucidated. Therefore, the present study evaluated LOXL2 expression in cellular compartments of the kidney and its possible contribution to tubulointerstitial fibrosis.

A previous study reported that LOXL2 expression in HK-2 cells was increased by hypoxia and hyperglycemia, and this alteration was associated with hypoxia inducible factor- $1 \alpha(21)$. The present study revealed that LOXL2 is primarily expressed in tubular cells in the kidney, particularly in distal tubular cells. There are numerous mechanisms by which tubular epithelial cells initiate or contribute to the progression of tubulointerstitial fibrosis. Following hypoxic, toxic or immunological insult-induced injury, tubular cells secrete chemoattractants to induce interstitial inflammation (22). TGF- $\beta 1$ and type III TGF- $\beta 1$ receptor have critical roles in the linkage of inflammation and fibrosis via the TGF- $\beta / \mathrm{Smad} 3$ signaling pathway (23). Epithelial-mesenchymal transition may also contribute to interstitial fibrosis by providing a source of fibrogenic myofibroblasts (24). Tubular epithelial cells lose cell-cell adhesion and acquire myofibroblast properties through the induction of TGF- $\beta$. Other signaling pathways and cellular components, including autophagy, Wnt/ $\beta$-catenin signaling and integrin-linked kinase, are also involved in this process $(25,26)$. 
A

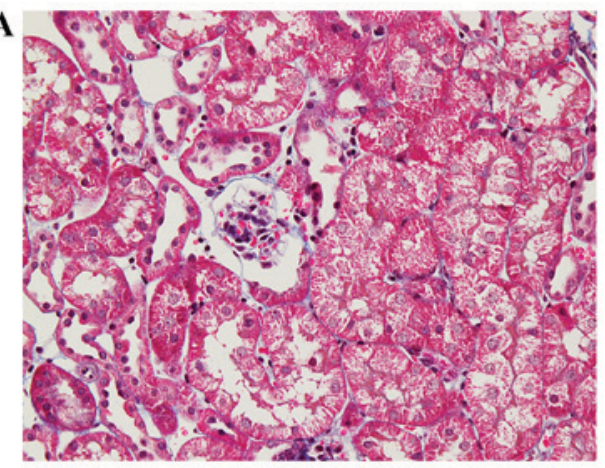

B
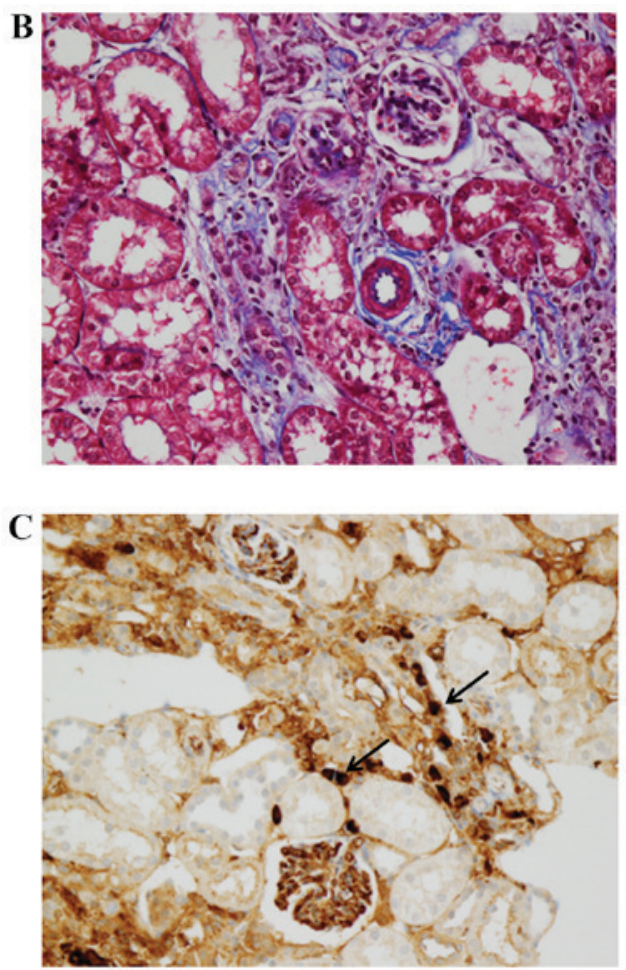

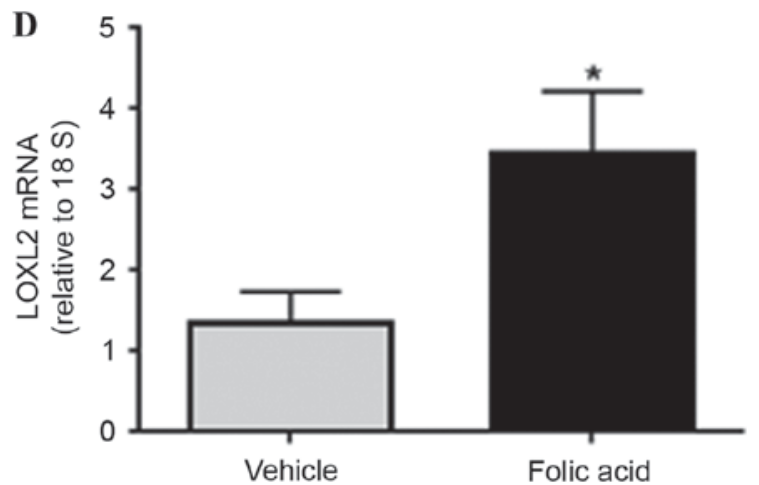

E

LOXL2

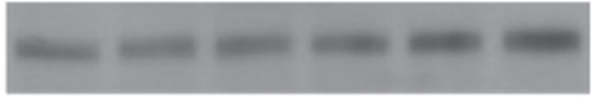

$\beta$-actin

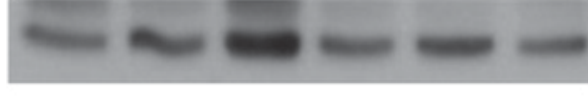

Vehicle

Folic acid

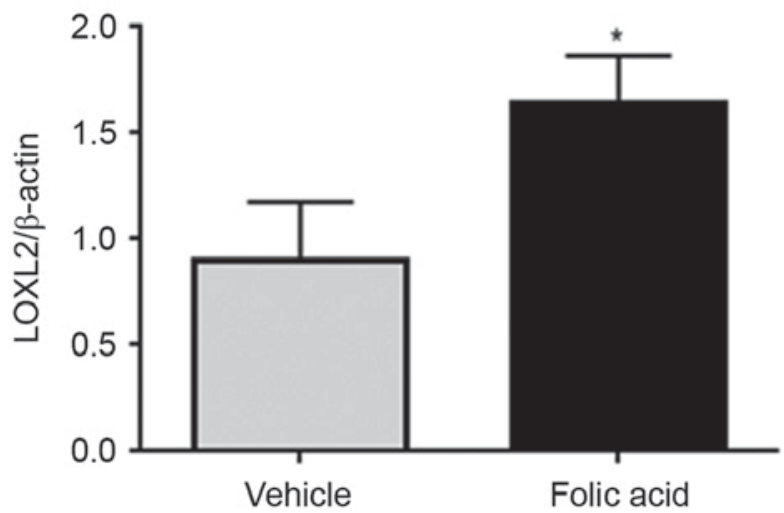

Figure 4. LOXL2 expression in a mouse model of tubulointerstitial fibrosis. Compared with in (A) vehicle-treated control mice, (B) diffuse tubulointerstitial fibrosis was induced 4 weeks after intraperitoneal injection of folic acid in CD1 mice. (C) Immunohistochemistry analysis detected LOXL2 protein expression in the fibrous interstitium and infiltrating mononuclear cells (arrows) in folic acid-injected mice. (D) mRNA expression levels of LOXL2 were markedly increased in folic acid-injected mice compared with vehicle-treated mice. (E) Results of a western blot analysis detected a significant increase in LOXL2 protein expression in folic acid-injected mice. Magnification, $\mathrm{x} 400$. ${ }^{*} \mathrm{P}<0.05$. LOXL2, lysyl oxidase-like 2.

Although the underlying mechanisms remain unclear, it is highly probable that tubular epithelial cells have a critical role in the progression of tubulointerstitial fibrosis. The present observation that LOXL2 is expressed in tubular epithelial cells suggests a role for LOXL2 in TGF- $\beta$-mediated tubulointerstitial fibrosis. This hypothesis is supported by the increased LOXL2 mRNA and protein levels detected in the kidneys of mice with folic acid-induced tubulointerstitial fibrosis.

The association between LOXL2 and TGF- $\beta$ has been investigated in numerous studies. Sethi et al demonstrated that the expression of LOX family genes, including LOXL2, is induced by TGF- $\beta 1$, TGF- $\beta 2$ and TGF- $\beta 3$, and is mediated by canonical Smad signaling and noncanonical signaling pathways (27). Voloshenyuk et al reported that TGF- $\beta 1$ upregulates LOX expression in cardiac fibroblasts, and this phenomenon may be prevented by inhibiting Smad3 (28). Conversely, an inhibitory monoclonal antibody against LOXL2 has been reported to decrease fibroblast activation and TGF- $\beta$ signaling, suggesting that LOXL2 serves a role in activating TGF- $\beta$ (13). Direct suppression of TGF- $\beta$ was not successful in preventing renal fibrosis due to of the diversity of TGF- $\beta$ isoforms and their signaling pathways (29). Therefore, it may be suggested that LOXL2, and other molecules involved in TGF- $\beta$ signaling pathways, should be considered as therapeutic targets.

The expression of LOXL2 in infiltrating inflammatory cells detected in the present study is interesting considering that infiltrating macrophages also express TGF- $\beta$ (30). This finding suggested that the potential mechanisms underlying the effects of LOXL2 in tubulointerstitial fibrosis are complex.

The present study also detected LOXL2 expression in podocytes, which may have clinical significance. Considerable evidence supports the role of podocyte injury as a key factor in the pathogenesis of focal segmental glomerulosclerosis (31-33). Podocyte detachment is also an important pathogenic mechanism in the progression of diabetic nephropathy (34), which is characterized by nodular glomerulosclerosis. Considering the 
profibrogenic function of LOXL2 in other organs, it is reasonable to hypothesize that podocyte LOXL2 may contribute to the progression of glomerulosclerosis.

In conclusion, LOXL2, which is a protein involved in extracellular matrix remodeling and organ fibrosis, is expressed in renal tubular epithelial cells and podocytes. Improved understanding regarding the function of LOXL2 in the kidney may strengthen knowledge of the pathophysiology of tubulointerstitial fibrosis and glomerulosclerosis, and may lead to the discovery of novel therapeutic targets.

\section{Acknowledgements}

The present study was supported by the Basic Science Research Program through the National Research Foundation of Korea funded by the Ministry of Science, ICT and Future Planning (grant no. NRF-2015R1C1A1A02036671).

\section{References}

1. Nishioka T, Eustace A and West C: Lysyl oxidase: From basic science to future cancer treatment. Cell Struct Funct 37: 75-80, 2012.

2. Fong SF, Dietzsch E, Fong KS, Hollosi P, Asuncion L, He Q, Parker MI and Csiszar K: Lysyl oxidase-like 2 expression is increased in colon and esophageal tumors and associated with less differentiated colon tumors. Genes Chromosomes Cancer 46 644-655, 2007.

3. Lin ZY, Chuang YH and Chuang WL: Cancer-associated fibroblasts up-regulate CCL2, CCL26, IL6 and LOXL2 genes related to promotion of cancer progression in hepatocellular carcinoma cells. Biomed Pharmacother 66: 525-529, 2012

4. Peinado H, Moreno-Bueno G, Hardisson D, Pérez-Gómez E, Santos V, Mendiola M, de Diego JI, Nistal M, Quintanilla M, Portillo F and Cano A: Lysyl oxidase-like 2 as a new poor prognosis marker of squamous cell carcinomas. Cancer Res 68 : 4541-4550, 2008.

5. Ahn SG, Dong SM, Oshima A, Kim WH, Lee HM, Lee SA, Kwon SH, Lee JH, Lee JM, Jeong J, et al: LOXL2 expression is associated with invasiveness and negatively influences survival in breast cancer patients. Breast Cancer Res Treat 141: 89-99, 2013.

6. Kasashima H, Yashiro M, Kinoshita H, Fukuoka T, Morisaki T, Masuda G, Sakurai K, Kubo N, Ohira M and Hirakawa K: Lysyl oxidase-like 2 (LOXL2) from stromal fibroblasts stimulates the progression of gastric cancer. Cancer Lett 354: 438-446, 2014

7. Barker HE, Bird D, Lang G and Erler JT: Tumor-secreted LOXL2 activates fibroblasts through FAK signaling. Mol Cancer Res 11: 1425-1436, 2013.

8. Barker HE, Chang J, Cox TR, Lang G, Bird D, Nicolau M, Evans HR, Gartland A and Erler JT: LOXL2-mediated matrix remodeling in metastasis and mammary gland involution. Cancer Res 71: 1561-1572, 2011.

9. Vadasz Z, Kessler O, Akiri G, Gengrinovitch S, Kagan HM, Baruch Y, Izhak OB and Neufeld G: Abnormal deposition of collagen around hepatocytes in Wilson's disease is associated with hepatocyte specific expression of lysyl oxidase and lysyl oxidase like protein-2. J Hepatol 43: 499-507, 2005.

10. Wong CC, Tse AP, Huang YP, Zhu YT, Chiu DK, Lai RK, Au SL, Kai AK, Lee JM, Wei LL, et al: Lysyl oxidase-like 2 is critical to tumor microenvironment and metastatic niche formation in hepatocellular carcinoma. Hepatology 60: 1645-1658, 2014.

11. Chien JW, Richards TJ, Gibson KF, Zhang Y, Lindell KO, Shao L, Lyman SK, Adamkewicz JI, Smith V, Kaminski N and O'Riordan T: Serum lysyl oxidase-like 2 levels and idiopathic pulmonary fibrosis disease progression. Eur Respir J 43: 1430-1438, 2014.

12. Van Bergen T, Marshall D, Van de Veire S, Vandewalle E, Moons L, Herman J, Smith V and Stalmans I: The role of LOX and LOXL2 in scar formation after glaucoma surgery. Invest Ophthalmol Vis Sci 54: 5788-5796, 2013.

13. Barry-Hamilton V, Spangler R, Marshall D, McCauley S, Rodriguez HM, Oyasu M, Mikels A, Vaysberg M, Ghermazien H, Wai C, et al: Allosteric inhibition of lysyl oxidase-like-2 impedes the development of a pathologic microenvironment. Nat Med 16 1009-1017, 2010
14. MeissnerEG, McLaughlin M, Matthews L, Gharib AM, Wood BJ Levy E, Sinkus R, Virtaneva K, Sturdevant D, Martens C, et al: Simtuzumab treatment of advanced liver fibrosis in HIV and HCV-infected adults: Results of a 6-month open-label safety trial. Liver Int 36: 1783-1792, 2016.

15. Saleem MA, O'Hare MJ, Reiser J, Coward RJ, Inward CD, Farren T, Xing CY, Ni L, Mathieson PW and Mundel P: A conditionally immortalized human podocyte cell line demonstrating nephrin and podocin expression. J Am Soc Nephrol 13: 630-638, 2002

16. Long DA, Woolf AS, Suda T and Yuan HT: Increased renal angiopoietin-1 expression in folic acid-induced nephrotoxicity in mice. J Am Soc Nephrol 12: 2721-2731, 2001.

17. Stallons LJ, Whitaker RM and Schnellmann RG: Suppressed mitochondrial biogenesis in folic acid-induced acute kidney injury and early fibrosis. Toxicol Lett 224: 326-332, 2014.

18. Livak KJ and Schmittgen TD: Analysis of relative gene expression data using real-time quantitative PCR and the 2(-Delta Delta C(T)) Method.. Methods 25: 402-408, 2001.

19. Meng XM, Nikolic-Paterson DJ and Lan HY: TGF-beta: The master regulator of fibrosis. Nat Rev Nephrol 12: 325-338, 2016.

20. Marek I, Lichtneger T, Cordasic N, Hilgers KF, Volkert G, Fahlbusch F, Rascher W, Hartner A and Menendez-Castro C: Alpha8 integrin (Itga8) signalling attenuates chronic renal interstitial fibrosis by reducing fibroblast activation, not by interfering with regulation of cell turnover. PLoS One 11: e0150471, 2016.

21. Sumual S, Saad S, Tang O, Yong R, McGinn S, Chen XM and Pollock CA: Differential regulation of Snail by hypoxia and hyperglycemia in human proximal tubule cells. Int J Biochem Cell Biol 42: 1689-1697, 2010.

22. Meng XM, Nikolic-Paterson DJ and Lan HY: Inflammatory processes in renal fibrosis. Nat Rev Nephrol 10: 493-503, 2014.

23. Meng XM, Huang XR, Xiao J, Chen HY, Zhong X, Chung AC and Lan HY: Diverse roles of TGF-b receptor II in renal fibrosis and inflammation in vivo and in vitro. J Pathol 227: 175-188, 2012.

24. Mack M and Yanagita M: Origin of myofibroblasts and cellular events triggering fibrosis. Kidney Int 87: 297-307, 2015.

25. Xiao L, Wang M, Yang S, Liu F and Sun L: A glimpse of the pathogenetic mechanisms of Wnt/b-catenin signaling in diabetic nephropathy. Biomed Res Int 2013: 987064, 2013.

26. Pang M, Wang H, Rao P, Zhao Y, Xie J, Cao Q, Wang Y, Wang YM, Lee VW, Alexander SI, et al: Autophagy links beta-catenin and Smad signaling to promote epithelial-mesenchymal transition via upregulation of integrin linked kinase. Int J Biochem Cell Biol 76: 123-134, 2016.

27. Sethi A, Mao W, Wordinger RJ and Clark AF: Transforming growth factor-beta induces extracellular matrix protein cross-linking lysyl oxidase (LOX) genes in human trabecular meshwork cells. Invest Ophthalmol Vis Sci 52: 5240-5250, 2011.

28. Voloshenyuk TG, Landesman ES, Khoutorova E, Hart AD and Gardner JD: Induction of cardiac fibroblast lysyl oxidase by TGF-b1 requires PI3K/Akt, Smad3, and MAPK signaling. Cytokine 55: 90-97, 2011.

29. Neelisetty S, Alford C, Reynolds K, Woodbury L, NlanduKhodo S, Yang H, Fogo AB, Hao CM, Harris RC, Zent R and Gewin L: Renal fibrosis is not reduced by blocking transforming growth factor-b signaling in matrix-producing interstitial cells. Kidney Int 88: 503-514, 2015.

30. Kim MG, Kim SC, Ko YS, Lee HY, Jo SK and Cho W: The role of M2 macrophages in the progression of chronic kidney disease following acute kidney injury. PLoS One 10: e0143961, 2015.

31. Chang JW, Pardo V, Sageshima J, Chen L, Tsai HL, Reiser J, Wei C, Ciancio G, Burke GW III and Fornoni A: Podocyte foot process effacement in postreperfusion allograft biopsies correlates with early recurrence of proteinuria in focal segmental glomerulosclerosis. Transplantation 93: 1238-1244, 2012.

32. Alachkar N, Wei C, Arend LJ, Jackson AM, Racusen LC, Fornoni A, Burke G, Rabb H, Kakkad K, Reiser J and Estrella MM: Podocyte effacement closely links to suPAR levels at time of posttransplantation focal segmental glomerulosclerosis occurrence and improves with therapy. Transplantation 96: 649-656, 2013.

33. Wharram BL, Goyal M, Wiggins JE, Sanden SK, Hussain S, Filipiak WE, Saunders TL, Dysko RC, Kohno K, Holzman LB and Wiggins RC: Podocyte depletion causes glomerulosclerosis: Diphtheria toxin-induced podocyte depletion in rats expressing human diphtheria toxin receptor transgene. J Am Soc Nephrol 16: 2941-2952, 2005.

34. Maezawa Y, Takemoto M and Yokote K: Cell biology of diabetic nephropathy: Roles of endothelial cells, tubulointerstitial cells and podocytes. J Diabetes Investig 6: 3-15, 2015. 